Ann. Funct. Anal. 6 (2015), no. 1, 148-169

http://doi.org/10.15352/afa/06-1-12

ISSN: 2008-8752 (electronic)

http://projecteuclid.org/afa

\title{
BANACH ALGEBRA TECHNIQUES TO COMPUTE SPECTRA, PSEUDOSPECTRA AND CONDITION SPECTRA OF SOME BLOCK OPERATORS WITH CONTINUOUS SYMBOLS
}

\author{
G. KRISHNA KUMAR AND S. H. KULKARNI \\ Communicated by M. S. Moslehian
}

ABSTRACT. In this paper we use Banach algebra techniques to study the spectrum, pseudospectrum and condition spectrum of a block Laurent operator with continuous symbol and a lower triangular block Toeplitz operator with continuous symbol.

(1) Let $L$ be a block Laurent operator with a continuous symbol $f$. Regarding $f$ as an element of the Banach algebra of all continuous matrix valued functions defined on the unit circle $\Gamma$, we show that the spectrum $\sigma(L)$ of $L$ coincides with the spectrum $\sigma(f)$ of $f$. It is also shown that the spectrum $\sigma(f)$ can be expressed as a union of the spectra of matrices $f(x)$. Thus

$$
\sigma(L)=\sigma(f)=\bigcup_{x \in \Gamma} \sigma(f(x))
$$

Similar results are proved about pseudospectrum

$$
\Lambda_{\epsilon}(L)=\Lambda_{\epsilon}(f)=\bigcup_{x \in \Gamma} \Lambda_{\epsilon}(f(x))
$$

for $\epsilon>0$ and condition spectrum $\sigma_{\epsilon}(L)=\sigma_{\epsilon}(f)$ for $0<\epsilon<1$.

(2) Let $T$ be an upper or lower triangular block Toeplitz operator with continuous symbol $f$. Then $f$ is a continuous matrix valued function defined on the closed unit disc $\Delta$ and $f$ is analytic in the open unit disc. It is proved that a similar description can be given about the spectrum $\sigma(T)$, pseudospectrum $\Lambda_{\epsilon}(T)$ for $\epsilon>0$ and condition spectrum $\sigma_{\epsilon}(T)$ for $0<\epsilon<1$.

These results are illustrated with examples and pictures using Matlab.

Date: Received: Feb. 17, 2013; Revised: Sep. 5, 2013; Accepted: Sep. 29, 2013.

2010 Mathematics Subject Classification. Primary 47B35; Secondary 47B48, 47A10, 46H35.

Key words and phrases. spectrum, pseudospectrum, condition spectrum, block operator, symbol. 


\section{INTRODUCTION}

Let $A$ be a complex unital Banach algebra with unit 1. For $\lambda \in \mathbb{C}$, we shall identify $\lambda .1$ with $\lambda$. We recall that the spectrum of an element $a \in A$ denoted by $\sigma(a, A)$ and is defined as

$$
\sigma(a, A)=\left\{\lambda \in \mathbb{C}: \lambda-a \notin A^{-1}\right\},
$$

where $A^{-1}$ denotes the set of all invertible elements of $A$. There are several generalizations of the concept of the spectrum in literature. The following are two important generalizations of spectrum.

Definition 1.1. ( $\epsilon$-pseudospectrum) Let $A$ be a complex unital Banach algebra with unit 1 and $\epsilon>0$. The $\epsilon$-pseudospectrum of an element $a \in A$ is denoted by $\Lambda_{\epsilon}(a, A)$ and is defined as,

$$
\Lambda_{\epsilon}(a, A):=\left\{\lambda \in \mathbb{C}:\left\|(\lambda-a)^{-1}\right\| \geq \frac{1}{\epsilon}\right\},
$$

with the convention that $\left\|(\lambda-a)^{-1}\right\|=\infty$ if $\lambda-a$ is not invertible.

Note that because of the above convention, $\sigma(a, A) \subseteq \Lambda_{\epsilon}(a, A)$ for every $\epsilon>0$. Pseudospectra provide an analytical and graphical alternative for investigating non-normal matrices and operators. For more information on $\epsilon$-pseudospectrum one may refer to [17].

Definition 1.2. ( $\epsilon$-condition spectrum) Let $A$ be a complex unital Banach algebra with unit 1 and $0<\epsilon<1$. The $\epsilon$-condition spectrum of an element $a \in A$ is denoted by $\sigma_{\epsilon}(a, A)$ and is defined as,

$$
\sigma_{\epsilon}(a, A):=\left\{\lambda \in \mathbb{C}:\|\lambda-a\|\left\|(\lambda-a)^{-1}\right\| \geq \frac{1}{\epsilon}\right\}
$$

with the convention that $\|\lambda-a\|\left\|(\lambda-a)^{-1}\right\|=\infty$, if $\lambda-a$ is not invertible.

Here also because of the above convention $\sigma(a, A) \subseteq \sigma_{\epsilon}(a, A)$ for $0<\epsilon<1$. One may refer to [11] for examples and elementary properties of the $\epsilon$-condition spectrum.

Definition 1.3. (inverse-closed subalgebra) Let $A$ be a complex unital Banach algebra with unit 1 and $B$ a unital subalgebra of $A$. We say that $B$ is inverseclosed in $A$ if every element of $B$, which is invertible in $A$, is also invertible in $B$, that is, $B \cap A^{-1} \subseteq B^{-1}$.

For more information on inverse-closed subalgebras one may refer to [14].

Theorem 1.4. Let $A$ be a complex unital Banach algebra with unit $1, B$ an inverse-closed subalgebra of $A$. Then for all $b \in B, \sigma(b, B)=\sigma(b, A)$. Also for all $\epsilon>0, \Lambda_{\epsilon}(b, B)=\Lambda_{\epsilon}(b, A)$ and for all $0<\epsilon<1, \sigma_{\epsilon}(b, B)=\sigma_{\epsilon}(b, A)$.

Proof. Clearly, for each $a \in B, \sigma(a, A) \subseteq \sigma(a, B)$. Next let $b \in B, \lambda \in \mathbb{C}$ and $\lambda \notin \sigma(b, A)$. Then $\lambda-b$ is invertible in $A$ and hence also invertible in $B$. Thus $\lambda \notin \sigma(b, B)$. Hence $\sigma(b, B) \subseteq \sigma(b, A)$. This shows that $\sigma(b, A)=\sigma(b, B)$. 
Since the norms in $B$ and $A$ are the same, it follows from the definition of pseudospectrum and condition spectrum that $\Lambda_{\epsilon}(b, B)=\Lambda_{\epsilon}(b, A)$ for all $\epsilon>0$ and $\sigma_{\epsilon}(b, B)=\sigma_{\epsilon}(b, A)$ for all $0<\epsilon<1$.

If the algebra under discussion is obvious we shall simplify the notation $\sigma(a, A)$ to $\sigma(a), \Lambda_{\epsilon}(a, A)$ to $\Lambda_{\epsilon}(a)$ and $\sigma_{\epsilon}(a, A)$ to $\sigma_{\epsilon}(a)$.

1.1. Block Laurent operator. Consider the standard $n$-dimensional vector space $\mathbb{C}^{m}$ with the Euclidean norm. If,

$$
x=\left(x_{1}, \cdots, x_{m}\right)
$$

is a vector in $\mathbb{C}^{m}$, then

$$
\|x\|_{2}=\left(\sum_{i=1}^{m}\left|x_{i}\right|^{2}\right)^{1 / 2}
$$

Let $\ell^{2}\left(\mathbb{Z}, \mathbb{C}^{m}\right)=\left\{x: \mathbb{Z} \rightarrow \mathbb{C}^{m}: \sum_{n \in \mathbb{Z}}\left\|x_{n}\right\|_{2}^{2}<\infty\right\}$. A bounded linear operator $L$ : $\ell^{2}\left(\mathbb{Z}, \mathbb{C}^{m}\right) \rightarrow \ell^{2}\left(\mathbb{Z}, \mathbb{C}^{m}\right)$ may be represented by a doubly infinite matrix,

$$
L=\left[A_{i j}\right]_{i, j=-\infty}^{\infty}
$$

where each entry is an operator on $\mathbb{C}^{m}$. Let $y=\left(y_{i}\right)_{i=-\infty}^{\infty}, x=\left(x_{i}\right)_{i=-\infty}^{\infty} \in$ $\ell^{2}\left(\mathbb{Z}, \mathbb{C}^{m}\right)$. Then $y=L x$ means

$$
y_{i}=\sum_{j \in \mathbb{Z}} A_{i j} x_{j}, \quad i \in \mathbb{Z}
$$

We call $L$ a block Laurent operator if its matrix elements $A_{i j}$ depend only on the difference $i-j$. The word block refers to the fact that the matrix entries are operators and not scalars. The doubly infinite matrix of the block Laurent operator $L$ has the following form.

$$
L=\left[\begin{array}{ccccccc}
\cdot & . & . & . & \ldots & . & . \\
\cdot & A_{1} & A_{0} & A_{-1} & \ldots & . & . \\
\cdot & \cdot & A_{1} & {\left[A_{0}\right]} & A_{-1} & . & . \\
\cdot & \cdot & \cdot & A_{1} & A_{0} & A_{-1} & \cdot \\
\cdot & \cdot & \ldots & \ldots & . & \cdot & .
\end{array}\right]
$$

$\left[A_{0}\right]$ denotes the $(0,0)$ entry which acts on the 0 -th coordinate space. Since $\ell^{2}\left(\mathbb{Z}, \mathbb{C}^{m}\right)$ is a direct sum of $m$ copies of $\ell^{2}(\mathbb{Z})$, an operator $L$ on $\ell^{2}\left(\mathbb{Z}, \mathbb{C}^{m}\right)$ may also be represented as an $m \times m$ matrix whose entries are operators acting on $\ell^{2}(\mathbb{Z})$. Thus

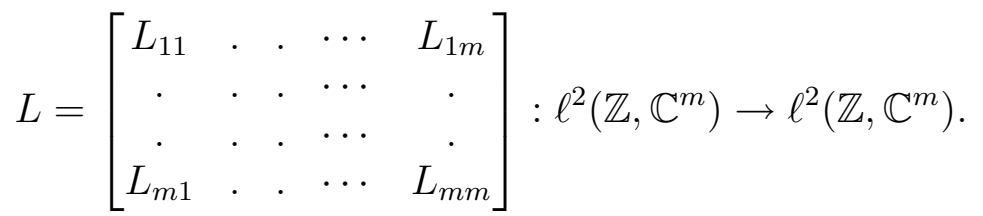

$L=\left[L_{i j}\right]_{i, j=1}^{m}$ is a block Laurent operator on $\ell^{2}\left(\mathbb{Z}, \mathbb{C}^{m}\right)$ if and only if each $L_{i j}$ is a Laurent operator on $\ell^{2}(\mathbb{Z})$. Let $L$ be a block Laurent operator on $\ell^{2}\left(\mathbb{Z}, \mathbb{C}^{m}\right)$ represented by the above two forms, then $L_{r s}=\left[A_{i-j}^{r s}\right]_{i, j=-\infty}^{\infty}$, where $A_{n}^{r s}$ is the 
$(r, s)$-th entry of the matrix $A_{n}$ with respect to the standard basis of $\mathbb{C}^{m}$. A brief discussion on block Laurent operators can be found in [8].

Let $\Gamma:=\{z \in \mathbb{C}:|z|=1\}$ denote the unit circle in the complex plane and $C(\Gamma)$ denote the set of all complex valued continuous functions on $\Gamma$. We shall use the symbol $C\left(\Gamma, \mathbb{C}^{m \times m}\right)$ to denote the set of all continuous matrix valued (i.e, $\mathbb{C}^{m \times m}$ valued) functions on $\Gamma$ (see Section 2 below). Note that $f \in C\left(\Gamma, \mathbb{C}^{m \times m}\right)$ can also be viewed as a matrix $f=\left[f_{i j}\right]_{m \times m}$ whose entries $f_{i j}$ belong to $C(\Gamma)$. For $f \in C(\Gamma)$, the $n$-th Fourier coefficient of $f$ is denoted by $f_{n}$ and is defined as $[7]$,

$$
f_{n}=\frac{1}{\sqrt{2 \pi}} \int_{-\pi}^{\pi} f(t) e^{-i n t} d t
$$

Let $\Phi \in C\left(\Gamma, \mathbb{C}^{m \times m}\right)$, then

$$
\Phi(t)=\left[\begin{array}{cccc}
\Phi_{11}(t) & \cdot & \cdots & \Phi_{1 m}(t) \\
\cdot & \cdot & \cdots & \cdot \\
\cdot & \cdot & \cdots & \cdot \\
\Phi_{m 1}(t) & \cdot & \cdots & \Phi_{m m}(t)
\end{array}\right]_{m \times m}
$$

where each $\Phi_{r s}$ is in $C(\Gamma)$. If the $n$-th Fourier coefficient of $\Phi_{r s}$ is equal to $A_{n}^{r s}$, the $r s^{\text {th }}$ entry of the matrix $A_{n}$, then

$$
\Phi_{r s}(t)=\sum_{n=-\infty}^{\infty} e^{i n t} A_{n}^{r s}
$$

If $L=\left[A_{i}\right]_{i=-\infty}^{\infty}$ is the block Laurent operator obtained from the continuous $m \times m$ matrix function $\Phi$, then $\Phi$ is called the continuous defining function or continuous symbol of the block Laurent operator $L$ (see [7], [8]).

1.2. Block Toeplitz operator. Let $\ell^{2}\left(\mathbb{N}, \mathbb{C}^{m}\right)=\left\{x: \mathbb{N} \rightarrow \mathbb{C}^{m}: \sum_{n=1}^{\infty}\left\|x_{n}\right\|_{2}^{2}<\infty\right\}$. A bounded linear operator $T: \ell^{2}\left(\mathbb{N}, \mathbb{C}^{m}\right) \rightarrow \ell^{2}\left(\mathbb{N}, \mathbb{C}^{m}\right)$ may be represented by an infinite matrix,

$$
T=\left[A_{i j}\right]_{i, j=1}^{\infty}
$$

whose entries are operators acting on $\mathbb{C}^{m}$. We call $T$ a block Toeplitz operator if its matrix elements $A_{i j}$ depend only on the difference $i-j$. Thus the infinite matrix of the block Toeplitz operator $T$ has the following form.

$$
T=\left[\begin{array}{cccc}
A_{0} & A_{-1} & A_{-2} & . . \\
A_{1} & A_{0} & A_{-1} & . . \\
A_{2} & A_{1} & A_{0} & . . \\
. . & . . & . . & . .
\end{array}\right]
$$

We call $T$ a lower triangular block Toeplitz operator if $A_{i}=[0]_{m \times m}$ for all $i<0$. Since $\ell^{2}\left(\mathbb{N}, \mathbb{C}^{m}\right)$ is a direct sum of $m$ copies of $\ell^{2}$, an operator $T$ on $\ell^{2}\left(\mathbb{N}, \mathbb{C}^{m}\right)$ 
may also be represented as an $m \times m$ matrix whose entries are operators acting on $\ell^{2}$. Thus

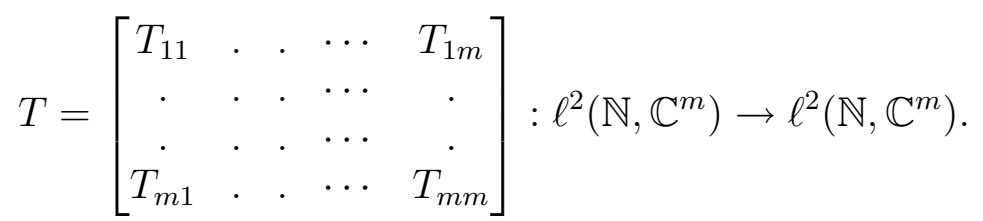

$T=\left[T_{i j}\right]_{i, j=1}^{m}$ is a block Toeplitz operator on $\ell^{2}\left(\mathbb{N}, \mathbb{C}^{m}\right)$, if and only if each $T_{i j}$ is a Toeplitz operator on $\ell^{2}$ [8]. The same can be said about the lower triangular block Toeplitz operators.

If $\left[T_{i-j}\right]_{i, j=0}^{\infty}$ is a block Toeplitz operator on $\ell^{2}\left(\mathbb{N}, \mathbb{C}^{m}\right)$, then $\left[T_{i-j}\right]_{i, j=-\infty}^{\infty}$ is a well defined block Laurent operator on $\ell^{2}\left(\mathbb{Z}, \mathbb{C}^{m}\right)$ and vice versa. A matrix valued function $\Phi$ on $\Gamma$ is called the defining function or symbol of the block Toeplitz operator $\left[T_{i-j}\right]_{i, j=0}^{\infty}$ if and only if $\Phi$ is the defining function of the block Laurent operator $\left[T_{i-j}\right]_{i, j=-\infty}^{\infty}[8]$. If $\Phi$ is a defining function of the lower triangular block Toeplitz operator, then

$$
\Phi(t)=\sum_{n=0}^{\infty} A_{j} t^{j}, \quad|t|=1 .
$$

A brief discussion on block Toeplitz operators is available in [8]. We use Banach algebra techniques to describe the spectra, pseudospectra and condition spectra of block Laurent operators with continuous symbols and lower(upper) triangular block Toeplitz operators with continuous symbols. Now we quote the theorem, proved in [10], which is essential for the same.

Theorem 1.5. Let $A, B$ be complex unital Banach algebras. Suppose $\Phi: A \rightarrow B$ is

(1) bijective,

(2) linear,

(3) multiplicative (that is, $\Phi(a b)=\Phi(a) \Phi(b)$ for all $a, b \in A$ ) or antimultiplicative (that is, $\Phi(a b)=\Phi(b) \Phi(a)$ for all $a, b \in A$ ) and

(4) isometry.

Then

$$
\begin{aligned}
\sigma(\Phi(a)) & =\sigma(a) \text { for all } a \in A \\
\Lambda_{\epsilon}(\Phi(a)) & =\Lambda_{\epsilon}(a) \text { for all } a \in A, \quad \epsilon>0, \text { and } \\
\sigma_{\epsilon}(\Phi(a)) & =\sigma_{\epsilon}(a) \text { for all } a \in A, \quad 0<\epsilon<1 .
\end{aligned}
$$

In this paper we attempt to describe the spectra, pseudospectra and condition spectra of block Laurent operators with continuous symbols and lower(upper) triangular block Toeplitz operators with continuous symbols in terms of their symbols using the theory of Banach algebra. In section 2, we introduce Banach algebra of continuous Banach algebra valued functions and discuss the spectrum, pseudospectrum and condition spectrum of an element in this algebra. In section 3, we describe the spectra, pseudospectra and condition spectra of block Laurent operators with continuous symbols. The main result here is that if $L$ is a block Laurent operator with a continuous symbol $f$, then the spectrum $\sigma(L)$ of $L$ 
coincides with the spectrum $\sigma(f)$ of $f$ (Theorem 3.2). Similarly $\Lambda_{\epsilon}(L)=\Lambda_{\epsilon}(f)$ for all $\epsilon>0$ and $\sigma_{\epsilon}(L)=\sigma_{\epsilon}(f)$ for all $0<\epsilon<1$ (Theorem 3.2). In section 4, we introduce the Banach algebra of Banach algebra valued analytic functions and discuss the spectrum, pseudospectrum and condition spectrum of an element in this algebra. In section 5, we describe the spectra, pseudospectra and condition spectra of a lower triangular block Toeplitz operators with continuous symbols. Again here the main result implies that the spectrum $\sigma(T)$ of a lower triangular Toeplitz operator with continuous symbol $f$, coincides with $\sigma(f)$ (Theorem 5.2). Also $\Lambda_{\epsilon}(T)=\Lambda_{\epsilon}(f)$ for all $\epsilon>0$ and $\sigma_{\epsilon}(T)=\sigma_{\epsilon}(f)$ for all $0<\epsilon<1$ (Theorem 5.2). Main results are illustrated with examples and pictures. Computations are done using matlab.

Some results on spectra and pseudospectra of Laurent operators with continuous symbols are available in [17]. Spectra of Toeplitz matrices and operators are discussed in [6]. The singular values of finite sections of block Toeplitz operators are discussed in [15]. Discrete spectra of some block Toeplitz operators are studied in [5]. Projection methods for block Toeplitz operators are investigated in [9].

\section{The Banach algebra $C(X, E)$}

Throughout this section, let $X$ denote a compact Hausdorff space, $(E,\|\cdot\|)$ be a complex unital Banach algebra with unit $e$ and $C(X, E)$ denote the space of all continuous $E$ valued functions on $X$. Then $C(X, E)$ is a complex Banach space under the norm,

$$
\|f\|_{\infty}=\sup \{\|f(x)\|: x \in X\}
$$

Since clearly,

$$
\|f g\|_{\infty} \leq\|f\|_{\infty}\|g\|_{\infty} \text { for all } f, g \in C(X, E)
$$

$C(X, E)$ is a complex unital Banach algebra with multiplication defined point wise. The unit element of $C(X, E)$ is denoted by 1 and is defined as,

$$
1(x)=e \text { for all } x \in X \text {. }
$$

If $E$ is commutative, then $C(X, E)$ is also commutative.

We consider an element $f \in C(X, E)$ and investigate the relationship between the spectrum $\sigma(f)$ of $f$ and the spectra $\sigma(f(x))$ for $x \in X$.

Theorem 2.1. An element $f \in C(X, E)$ is invertible if and only if $f(x)$ is invertible in $E$ for all $x \in X$ and in this case $f^{-1}(x)=f(x)^{-1}$ for all $x \in X$. Consequently,

$$
\sigma(f)=\bigcup_{x \in X} \sigma(f(x)) \text { for all } f \in C(X, E) .
$$

Proof. Proof is elementary and depends on the following observation: If for $f \in$ $C(X, E) f(x)$ is invertible in $E$ for all $x \in X$, then the function $g: X \rightarrow E$ defined by ,

$$
g(x)=f(x)^{-1} \quad \text { for all } x \in X
$$

is continuous. 
Next we consider the pseudospectrum $\Lambda_{\epsilon}(f)$ of $f \in C(X, E)$ and pseudospectra $\Lambda_{\epsilon}(f(x))$ for $x \in X$ and show that a similar relation exists among these sets.

Theorem 2.2. Let $f \in C(X, E)$ and $\epsilon>0$. Then

$$
\Lambda_{\epsilon}(f)=\bigcup_{x \in X} \Lambda_{\epsilon}(f(x)) \text { for all } f \in C(X, E) .
$$

Proof. Let $\lambda \in \Lambda_{\epsilon}(f)$, if $\lambda \in \sigma(f)$ then by Theorem 2.1,

$$
\lambda \in \bigcup_{x \in X} \sigma(f(x)) \subseteq \bigcup_{x \in X} \Lambda_{\epsilon}(f(x)) .
$$

If $\lambda \in \Lambda_{\epsilon}(f) \backslash \sigma(f)$, then $\lambda-f$ is invertible and

$$
\left\|(\lambda-f)^{-1}\right\|_{\infty} \geq \frac{1}{\epsilon}
$$

Since $X$ is compact, there exists $x_{0} \in X$ such that,

$$
\left\|(\lambda-f)^{-1}\right\|_{\infty}=\left\|(\lambda-f)^{-1} x_{0}\right\|=\left\|\left(\lambda-f\left(x_{0}\right)\right)^{-1}\right\| \geq \frac{1}{\epsilon} .
$$

So,

$$
\lambda \in \Lambda_{\epsilon}\left(f\left(x_{0}\right)\right) \subseteq \bigcup_{x \in X} \Lambda_{\epsilon}(f(x)) .
$$

Thus,

$$
\Lambda_{\epsilon}(f) \subseteq \bigcup_{x \in X} \Lambda_{\epsilon}(f(x))
$$

To prove the reverse inclusion consider $\lambda \in \bigcup_{x \in X} \Lambda_{\epsilon}(f(x))$. Then $\lambda \in \Lambda_{\epsilon}\left(f\left(x_{0}\right)\right)$ for some $x_{0} \in X$. Thus,

$$
\left\|\left(\lambda-f\left(x_{0}\right)\right)^{-1}\right\| \geq \frac{1}{\epsilon} .
$$

If $\lambda \in \sigma(f(x))$ for some $x \in X$, then $\lambda \in \sigma(f) \subseteq \Lambda_{\epsilon}(f)$ by Theorem 2.1. So consider the case when $\lambda \notin \sigma(f(x))$ for all $x \in X$. Then $\lambda \notin \sigma(f)$ and

$$
\left\|(\lambda-f)^{-1}\right\|_{\infty} \geq\left\|\left(\lambda-f\left(x_{0}\right)\right)^{-1}\right\| \geq \frac{1}{\epsilon} .
$$

Thus $\lambda \in \Lambda_{\epsilon}(f)$. Hence

$$
\bigcup_{x \in X} \Lambda_{\epsilon}(f(x)) \subseteq \Lambda_{\epsilon}(f) .
$$

These two inclusions prove the desired result.

Finally we attempt to obtain a similar relationship between the condition spectrum $\sigma_{\epsilon}(f)$ of $f \in C(X, E)$ and $\sigma_{\epsilon}(f(x))$ for $x \in X$. Here the relations are more complicated. In the following $D(z, r)$ denotes the closed disk in the complex plane with center $z$ and radius $r>0$. 
Theorem 2.3. Let $f \in C(X, E)$ and $0<\epsilon<1$. Then

$\bigcup_{x \in X} \sigma_{\epsilon}(f(x)) \subseteq \sigma_{\epsilon}(f) \subseteq \bigcup_{x \in X}\left\{D\left(\alpha, \epsilon_{1}\right): f(x)=\alpha e\right\} \bigcup_{x \in X}\left\{\sigma_{\frac{\epsilon_{1}}{m_{x}}}(f(x)): m_{x}>0\right\}$

where $\epsilon_{1}=\frac{2 \epsilon}{1-\epsilon}\|f\|_{\infty}$ and $m_{x}=\inf \{\|z-f(x)\|: z \in \mathbb{C}\}$.

Proof. Let $\lambda \in \bigcup_{x \in X} \sigma_{\epsilon}(f(x))$. If $\lambda \in \sigma(f(x))$ for some $x \in X$, then by Theorem 2.1, $\lambda \in \sigma(f) \subseteq \sigma_{\epsilon}(f)$. So consider the case when $\lambda \notin \sigma(f(x))$ for all $x \in X$. Then by Theorem 2.1, $\lambda \notin \sigma(f)$. Since $\lambda \in \sigma_{\epsilon}\left(f\left(x_{0}\right)\right)$ for some $x_{0} \in X$

$$
\|\lambda-f\|_{\infty}\left\|(\lambda-f)^{-1}\right\|_{\infty} \geq\left\|\lambda-f\left(x_{0}\right)\right\|\left\|\left(\lambda-f\left(x_{0}\right)\right)^{-1}\right\| \geq \frac{1}{\epsilon} .
$$

Hence $\lambda \in \sigma_{\epsilon}(f)$. Next let $\lambda \in \sigma_{\epsilon}(f)$. If $\lambda \in \sigma(f)$, then $\lambda \in \sigma(f(x))$ and hence $\lambda \in \bigcup_{x \in X}\left\{D\left(\alpha, \epsilon_{1}\right): f(x)=\alpha e\right\} \bigcup_{x \in X}\left\{\sigma_{\frac{\epsilon_{1}}{m_{x}}}(f(x)): m_{x}>0\right\}$. Suppose $\lambda \in$ $\sigma_{\epsilon}(f) \backslash \sigma(f)$. Then

$$
\begin{aligned}
\frac{1}{\epsilon} & \leq\|\lambda-f\|_{\infty}\left\|(\lambda-f)^{-1}\right\|_{\infty} \\
& \leq\left\|(\lambda-f)^{-1}\right\|_{\infty}\left(|\lambda|+\|f\|_{\infty}\right) \\
& \leq\left\|(\lambda-f)^{-1}\right\|_{\infty} \frac{2\|f\|_{\infty}}{1-\epsilon} .
\end{aligned}
$$

Let $\epsilon_{1}=\frac{2 \epsilon}{1-\epsilon}\|f\|_{\infty}$. Then $\left\|(\lambda-f)^{-1}\right\|_{\infty} \geq \frac{1}{\epsilon_{1}}$ and there exist $x \in X$ such that $\left\|(\lambda-f)^{-1}\right\|_{\infty}=\left\|(\lambda-f(x))^{-1}\right\|$. Thus $\left\|(\lambda-f(x))^{-1}\right\| \geq \frac{1}{\epsilon_{1}}$. Now if $f(x)=\alpha e$ for some $\alpha \in \mathbb{C}$, then $\left\|(\lambda-f(x))^{-1}\right\|=\frac{1}{|\lambda-\alpha|}$. Thus $|\lambda-\alpha| \leq \epsilon_{1}$. Otherwise, let $m_{x}=\inf \{\|z-f(x)\|: z \in \mathbb{C}\}$. Then $0<m_{x}<\|\lambda-f(x)\|$. Hence

$$
\frac{m_{x}}{\epsilon_{1}} \leq\|\lambda-f(x)\|\left\|(\lambda-f(x))^{-1}\right\| .
$$

Thus $\lambda \in \sigma_{\frac{\epsilon_{1}}{m_{x}}}(f(x))$.

Remark 2.4. The first inclusion in the above theorem can be strict. Consider Example 2.10 in [11], $X=[-1,1], E=\mathbb{C}$ and $f(x)=x$ for $x$ in $[-1,1]$. Then $\sigma_{\epsilon}(f(x))=f(x)$. So $\bigcup_{x \in X} \sigma_{\epsilon}(f(x))=[-1,1]$. On the other hand $\lambda=\frac{1+\epsilon}{1-\epsilon} \in \sigma_{\epsilon}(f)$, but $\lambda \notin \sigma_{\epsilon}(f(x))$ for any $x \in X$.

\section{Block Laurent operators With CONTINUOUS Symbols}

In this section, using the theory developed in the last section, we describe the spectrum, pseudospectrum and condition spectrum of a block Laurent operator 
with continuous symbol. For this purpose, we take $X=\Gamma$, the unit circle in $\mathbb{C}$, and $E=\left(\mathbb{C}^{m \times m},\|\cdot\|_{2}\right)$. We have for $M \in E$,

$$
\|M\|_{2}=\sup \left\{\|M x\|_{2}: x \in \mathbb{C}^{m} \text { and }\|x\|_{2} \leq 1\right\} \text {. }
$$

For a Banach space $E, B L(E)$ denotes the Banach algebra of all bounded linear operators on $E$ with usual operations and the operator norm.

Theorem 3.1. Let $A$ be the Banach algebra of all block Laurent operators on $\ell^{2}\left(\mathbb{Z}, \mathbb{C}^{m}\right)$ with continuous symbols. Then $A$ is inverse-closed in $B L\left(\ell^{2}\left(\mathbb{Z}, \mathbb{C}^{m}\right)\right)$.

Proof. Let $S$ denote the forward shift operator on $\ell^{2}\left(\mathbb{Z}, \mathbb{C}^{m}\right)$ and $L \in B L\left(\ell^{2}\left(\mathbb{Z}, \mathbb{C}^{m}\right)\right)$. A known result says that $L$ is a block Laurent operator if and only if $L S=S L$ [8]. Let $L \in B L\left(\ell^{2}\left(\mathbb{Z}, \mathbb{C}^{m}\right)\right)$ be an invertible block Laurent operator with a continuous symbol $\Phi(t), t \in \Gamma$. Then $L S=S L$, hence

$$
L^{-1} S=L^{-1} S\left(L L^{-1}\right)=L^{-1}(S L) L^{-1}=L^{-1}(L S) L^{-1}=S L^{-1} .
$$

Thus $L^{-1}$ is a block Laurent operator. Further since $L$ is invertible $\Phi(t) \neq 0$ for all $t \in \Gamma$ and $\Phi(t)^{-1}, t \in \Gamma$ is the symbol of $L^{-1}$. Since $\Phi(t) \in C\left(\Gamma, \mathbb{C}^{m \times m}\right)$ and $\Phi(t) \neq 0$ for all $t \in \Gamma$ we have $\Phi(t)^{-1} \in C\left(\Gamma, \mathbb{C}^{m \times m}\right)$. Hence $L^{-1} \in A$.

Theorem 3.2. Let $L$ be a block Laurent operator on $\ell^{2}\left(\mathbb{Z}, \mathbb{C}^{m}\right)$ with a continuous symbol $f \in C\left(\Gamma, \mathbb{C}^{m \times m}\right)$. Then $\sigma(L)=\sigma(f)$. Also for each $\epsilon>0, \Lambda_{\epsilon}(L)=$ $\Lambda_{\epsilon}(f)$ and for $0<\epsilon<1, \sigma_{\epsilon}(L)=\sigma_{\epsilon}(f)$, where $f$ is regarded as an element of $C\left(\Gamma, \mathbb{C}^{m \times m}\right)$.

Proof. Let $A$ be the Banach algebra of all block Laurent operators on $\ell^{2}\left(\mathbb{Z}, \mathbb{C}^{m}\right)$ with continuous symbols and $B=C\left(\Gamma, \mathbb{C}^{m \times m}\right)$. From Theorem 3.1, $A$ is inverse closed in $B L\left(\ell^{2}\left(\mathbb{Z}, \mathbb{C}^{m}\right)\right)$. Hence by Theorem 1.4, the spectrum of $L$ as an element of $A$ is the same as the spectrum of $L$ regarded as an element of $B L\left(\ell^{2}\left(\mathbb{Z}, \mathbb{C}^{m}\right)\right)$. The same can be said about the pseudospectrum and condition spectrum. The map $\Phi: A \rightarrow B$ defined by,

$$
\Phi(L)=\text { symbol of } L
$$

is linear, bijective, unital, multiplicative (Lemma 4.1, page 575) and isometry (corollary 2.2, page 567) [8]. Hence the result follows from Theorem 1.5.

Corollary 3.3. Let $L$ be a block Laurent operator on $\ell^{2}\left(\mathbb{Z}, \mathbb{C}^{m}\right)$ with continuous symbol $f=\left[f_{k l}\right]_{k, l=1}^{m} \in C\left(\Gamma, \mathbb{C}^{m \times m}\right)$. Then

$$
\begin{aligned}
\text { 1. } \sigma(L) & =\bigcup_{x \in \Gamma} \sigma(f(x))=\bigcup_{x \in \Gamma} \sigma\left(\left[f_{k l}(x)\right]_{k, l=1}^{m}\right) . \\
\text { 2. } \Lambda_{\epsilon}(L) & =\bigcup_{x \in \Gamma} \Lambda_{\epsilon}(f(x))=\bigcup_{x \in \Gamma} \Lambda_{\epsilon}\left(\left[f_{k l}(x)\right]_{k, l=1}^{m}\right) . \\
\text { 3. } \sigma_{\epsilon}(L) & \supseteq \bigcup_{x \in \Gamma} \sigma_{\epsilon}(f(x))=\bigcup_{x \in \Gamma} \sigma_{\epsilon}\left(\left[f_{k l}(x)\right]_{k, l=1}^{m}\right) . \\
\text { 4. } \sigma_{\epsilon}(L) & \subseteq \bigcup_{x \in \Gamma}\left\{D\left(\alpha, \epsilon_{1}\right):\left[f_{k l}(x)\right]_{k, l=1}^{m}=\alpha I_{m}\right\} \bigcup_{x \in \Gamma}\left\{\sigma_{\frac{\epsilon_{1}}{m_{x}}}\left(\left[f_{k l}(x)\right]_{k, l=1}^{m}\right): m_{x}>0\right\} .
\end{aligned}
$$


where $I_{m}$ is the $m \times m$ identity matrix, $\epsilon_{1}=\frac{2 \epsilon}{1-\epsilon}\|f\|_{\infty}$ and for $x \in \Gamma, m_{x}=$ $\inf \{\|z-f(x)\|: z \in \mathbb{C}\}$.

Proof. (1) From Theorem 3.2 and Theorem 2.1 we have

$$
\sigma(L)=\sigma(f)=\bigcup_{x \in \Gamma} \sigma(f(x)) .
$$

Since $f \in C\left(\Gamma, \mathbb{C}^{m \times m}\right), f=\left[f_{k l}\right]_{k, l=1}^{m}$ such that each $f_{k, l} \in C(\Gamma)$. Hence

$$
\bigcup_{x \in \Gamma} \sigma(f(x))=\bigcup_{x \in \Gamma} \sigma\left(\left[f_{k, l}(x)\right]_{k, l=1}^{m}\right)
$$

(2) From Theorem 3.2 and Theorem 2.2 we have

$$
\Lambda_{\epsilon}(L)=\Lambda_{\epsilon}(f)=\bigcup_{x \in \Gamma} \Lambda_{\epsilon}(f(x))=\bigcup_{x \in \Gamma} \Lambda_{\epsilon}\left(\left[f_{k, l}(x)\right]_{k, l=1}^{m}\right) .
$$

(3) From Theorem 3.2 and Theorem 2.3 we have

$$
\sigma_{\epsilon}(L)=\sigma_{\epsilon}(f) \supseteq \bigcup_{x \in \Gamma} \sigma_{\epsilon}(f(x))=\bigcup_{x \in \Gamma} \sigma_{\epsilon}\left(\left[f_{k, l}(x)\right]_{k, l=1}^{m}\right) .
$$

(4) From Theorem 3.2 and Theorem 2.3 we have

$$
\sigma_{\epsilon}(L)=\sigma_{\epsilon}(f) \subseteq \bigcup_{x \in \Gamma}\left\{D\left(\alpha, \epsilon_{1}\right): f(x)=\alpha I_{m}\right\} \bigcup_{x \in \Gamma}\left\{\sigma_{\frac{\epsilon_{1}}{m_{x}}}(f(x)): m_{x}>0\right\}
$$

where $\epsilon_{1}=\frac{2 \epsilon}{1-\epsilon}\|f\|_{\infty}$ and for $x \in \Gamma, m_{x}=\inf \{\|z-f(x)\|: z \in \mathbb{C}\}$. We note that the right hand side is equal to

$$
\bigcup_{x \in \Gamma}\left\{D\left(\alpha, \epsilon_{1}\right):\left[f_{k, l}(x)\right]_{k, l=1}^{m}=\alpha I_{m}\right\} \bigcup_{x \in \Gamma}\left\{\sigma_{\frac{\epsilon_{1}}{m_{x}}}\left(\left[f_{k, l}(x)\right]_{k, l=1}^{m}\right): m_{x}>0\right\}
$$

Corollary 3.4. Let $L$ be a Laurent operator on $\ell^{2}(\mathbb{Z})$ with a continuous symbol $f \in C(\Gamma)$. Then

1. $\sigma(L)=\{f(x): x \in \Gamma\}=f(\Gamma)$.

2. $\Lambda_{\epsilon}(L)=\bigcup\{D(f(x), \epsilon): x \in \Gamma\}$.

3. $\sigma_{\epsilon}(L) \supseteq\{f(x): x \in \Gamma\}$.

4. $\sigma_{\epsilon}(L) \subseteq \bigcup\left\{D\left(f(x), \frac{2 \epsilon}{1-\epsilon}\|f\|_{\infty}\right): x \in \Gamma\right\}$.

Proof. (1) From Corollary 3.3

$$
\sigma(L)=\sigma(f)=\bigcup_{x \in \Gamma} \sigma(f(x))=\bigcup_{x \in \Gamma} f(x)=f(\Gamma) .
$$


(2) From Corollary 3.3

$$
\Lambda_{\epsilon}(L)=\Lambda_{\epsilon}(f)=\bigcup_{x \in \Gamma} \Lambda_{\epsilon}(f(x))=\bigcup_{x \in \Gamma} D(f(x), \epsilon)
$$

(3) From Corollary 3.3

$$
\sigma_{\epsilon}(L)=\sigma_{\epsilon}(f) \supseteq \bigcup_{x \in \Gamma} \sigma_{\epsilon}(f(x))=\bigcup_{x \in \Gamma}\{f(x)\}=f(\Gamma) .
$$

(4) From Corollary 3.3

$$
\sigma_{\epsilon}(L)=\sigma_{\epsilon}(f) \subseteq \bigcup_{x \in \Gamma} D\left(f(x), \frac{2 \epsilon}{1-\epsilon}\|f\|_{\infty}\right)
$$

Remark 3.5. Theorem 3.2 and its corollaries are useful in computing spectrum $\sigma(L)$, pseudospectrum $\Lambda_{\epsilon}(L)$ and condition spectrum $\sigma_{\epsilon}(L)$ of a block Laurent operator $L$ with a continuous symbol $f \in C\left(\Gamma, \mathbb{C}^{m \times m}\right)$. Since for each $x \in \Gamma, f(x)$ is a matrix of order $m \times m$, we can compute and plot $\sigma(f(x)), \Lambda_{\epsilon}(f(x)), \sigma_{\epsilon}(f(x))$ for various values of $x \in \Gamma$. Then the respective unions of these sets are approximations of $\sigma(L), \Lambda_{\epsilon}(L), \sigma_{\epsilon}(L)$.

The situation is very satisfactory when $m=1$, that is, $L$ is a Laurent operator on $\ell^{2}(\mathbb{Z})$. From Corollary 3.4, $\sigma(L)=f(\Gamma)$ is a closed curve in the complex plane, say $\gamma_{0}$. Let $\gamma_{1}$ and $\gamma_{2}$ be the closed curves lying on either side of $\gamma_{0}$ and parallel to $\gamma_{0}$ at a distance $\epsilon$ from $\gamma_{0}$. Then $\Lambda_{\epsilon}(L)$ is the region lying between $\gamma_{1}$ and $\gamma_{2} . \sigma_{\epsilon}(L)$ is also contained in a similar region. From Theorem 3.2, $\sigma_{\epsilon}(L)=\sigma_{\epsilon}(f)$. We can consider certain number of uniformly distributed points in the $\operatorname{disc}\left\{z \in \mathbb{C}:|z| \leq \frac{1+\epsilon}{1-\epsilon}\|f\|_{\infty}\right\}[11]$, evaluate $\|z-f\|_{\infty}\left\|(z-f)^{-1}\right\|_{\infty}$ at each of these points and include and save those $z$ for which $\|z-f\|_{\infty}\left\|(z-f)^{-1}\right\|_{\infty} \geq \frac{1}{\epsilon}$. For example, if $L$ is the right shift operator, then $f(z)=z, \sigma(L)=\Gamma, \Lambda_{\epsilon}(L)$ is the annulus $\{\lambda \in \mathbb{C}: 1-\epsilon \leq|\lambda| \leq 1+\epsilon\}$ (see Theorem 3.3 of [10]), $\sigma_{\epsilon}(L)$ is the annulus $\left\{\lambda \in \mathbb{C}: \frac{1-\epsilon}{1+\epsilon} \leq|\lambda| \leq \frac{1+\epsilon}{1-\epsilon}\right\}$ (see Theorem 3.3 of [10]).

In the following examples the pseudospectra and condition spectra of matrices are calculated using the basic algorithm explained in [17]. There are many efficient algorithms to compute the pseudospectra of matrices [17]. Some of these can be modified to compute the condition spectra also. Since our aim is only to illustrate the above results, we do not make any claim about the efficiency of this algorithm. 
Example 3.6. Consider the block Laurent operator

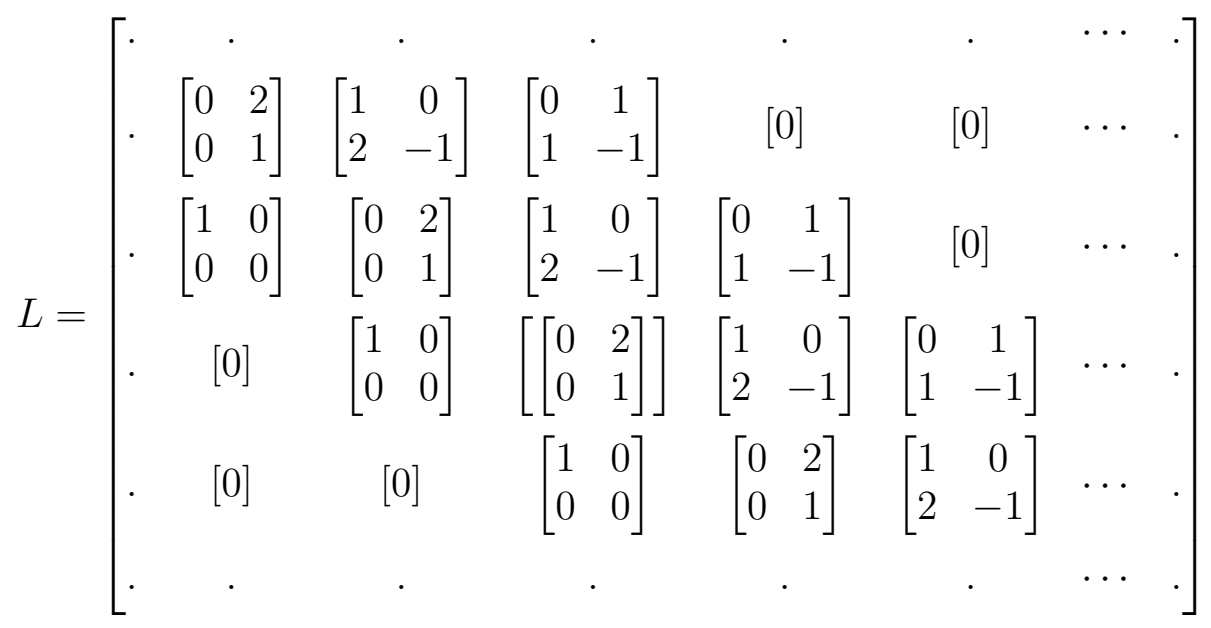

where [0] denotes the $2 \times 2$ zero matrix. Let $f$ denote the symbol of $L$. Then

$$
f(x)=\left[\begin{array}{ll}
f_{11}(x) & f_{12}(x) \\
f_{21}(x) & f_{22}(x)
\end{array}\right]=\left[\begin{array}{cc}
x+\frac{1}{x} & 2+\frac{1}{x^{2}} \\
\frac{2}{x}+\frac{1}{x^{2}} & 1-\frac{1}{x}-\frac{1}{x^{2}}
\end{array}\right], \quad x \in \Gamma .
$$

By Corollary 3.3, $\sigma(L)$ is the union of the eigenvalues of the matrix $f(x)$ for $x \in \Gamma$. The $\epsilon$-pseudospectrum and $\epsilon$-condition spectrum of $L$ is calculated using Remark 3.5. The following figures are obtained using matlab. Figure 1.1 shows $\sigma(L)$, Figure 1.2 shows $\Lambda_{0.5}(L)$, Figure 1.3( $\left.D_{1}\right)$ shows the set defined in (3) of Corollary 3.3 and Figure 1.4 $\left(D_{2}\right)$ shows the set defined in (4) of Corollary 3.3 for $\epsilon=0.01$. We have $D_{1} \subseteq \sigma_{0.01}(L) \subseteq D_{2}$, Figure 1.5 shows $\sigma_{0.01}(L)$.
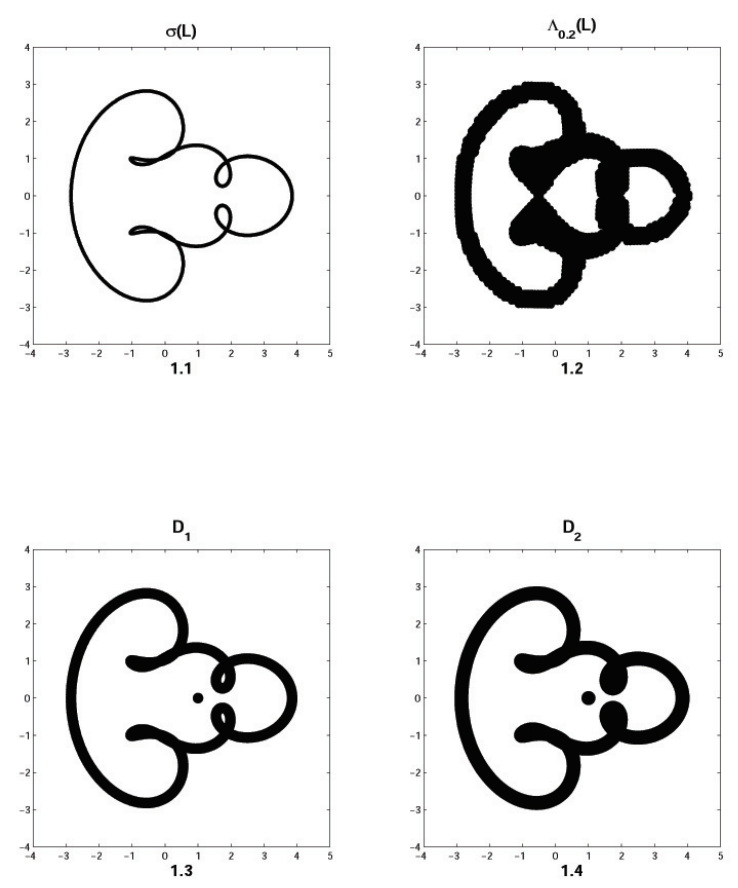


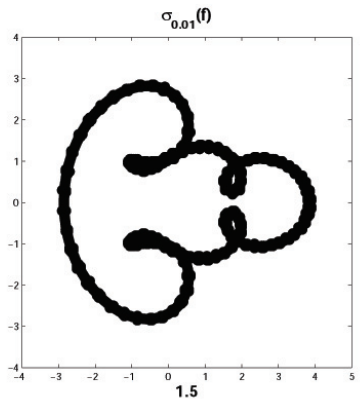

Example 3.7. Consider the block Laurent operator

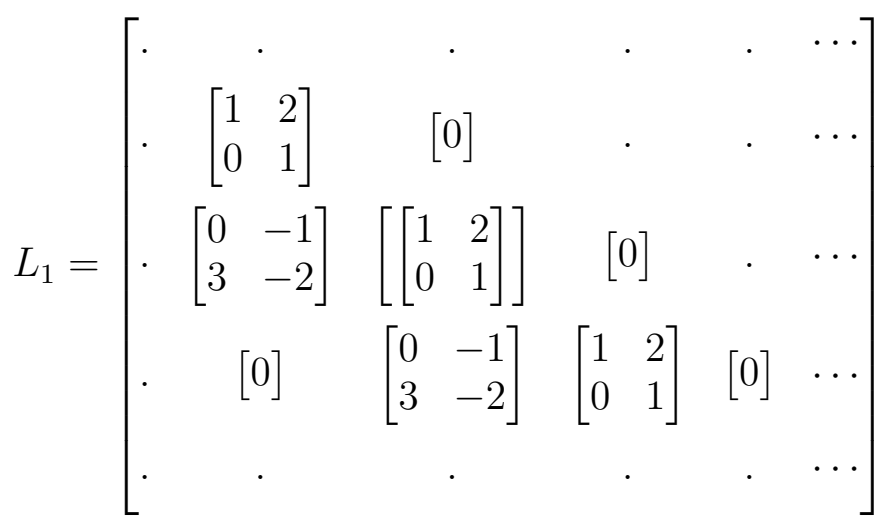

where [0] denotes the $2 \times 2$ zero matrix. Let $f$ denote the symbol of $L_{1}$. Then

$$
f(x)=\left[\begin{array}{ll}
f_{11}(x) & f_{12}(x) \\
f_{21}(x) & f_{22}(x)
\end{array}\right]=\left[\begin{array}{cc}
1 & 2-x \\
3 x & 1-2 x
\end{array}\right], \quad x \in \Gamma .
$$

Figure 2.1 shows $\sigma\left(L_{1}\right)$, Figure 2.2 shows $\Lambda_{0.5}\left(L_{1}\right)$, Figure $2.3\left(D_{1}\right)$ shows the set defined in (3) of Corollary 3.3 and Figure 2.4 $\left(D_{2}\right)$ shows the set defined in (4) of Corollary 3.3 for $\epsilon=0.01$. We have $D_{1} \subseteq \sigma_{0.01}\left(L_{1}\right) \subseteq D_{2}$, Figure 1.5 shows $\sigma_{0.01}\left(L_{1}\right)$.
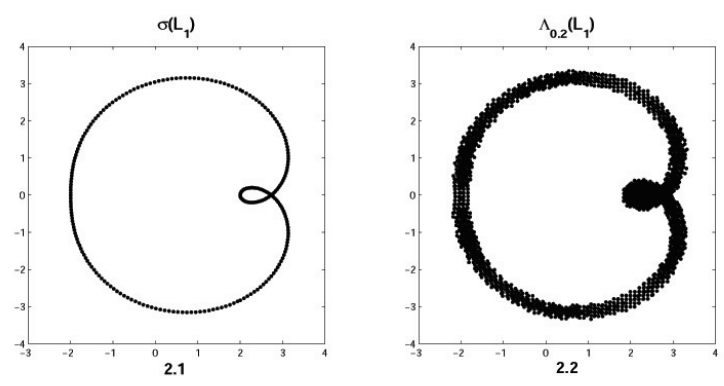

\section{Banach ALgebra $H(\Delta, E)$}

Throughout this section let $(E,\|\cdot\|)$ be a complex Banach algebra with unit $e$, let $\Delta$ denote the closed unit disk of the complex plane and $\Delta^{0}$ denote the interior 

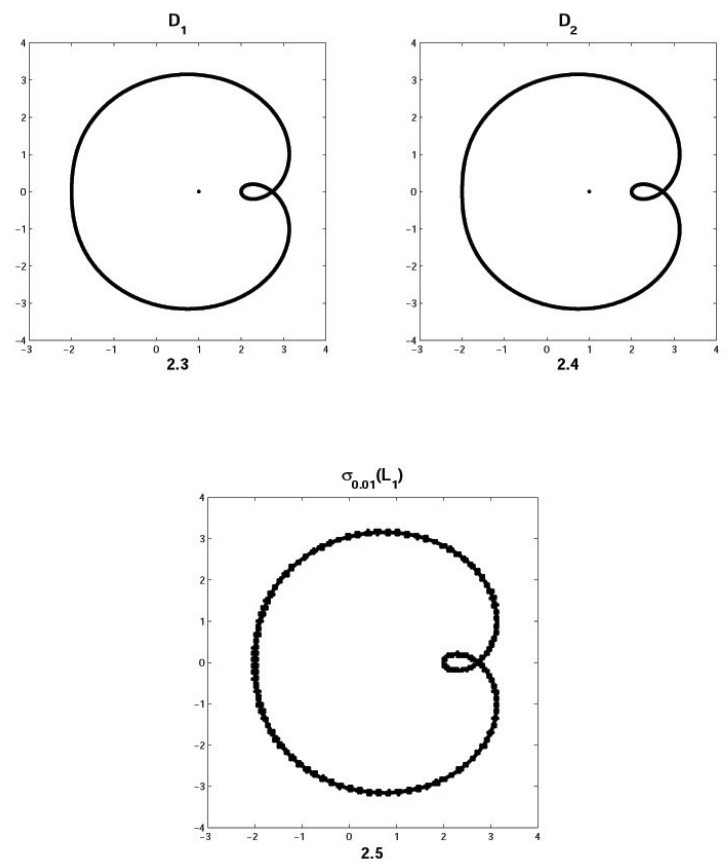

of $\Delta$. A function $f: \Delta \rightarrow E$ is said to be differentiable at $z_{0} \in \Delta^{0}$ if

$$
f^{\prime}\left(z_{0}\right)=\lim _{z \rightarrow z_{0}} \frac{f(z)-f\left(z_{0}\right)}{z-z_{0}}
$$

exists (in the norm on $E$ ). If $f$ is differentiable at all points of $\Delta^{0}$, we say that $f$ is analytic in $\Delta^{0}$. Define

$$
H(\Delta, E)=\left\{f \in C(\Delta, E):\left.f\right|_{\Delta^{0}} \text { is analytic in } \Delta^{0}\right\} .
$$

Let $f \in H(\Delta, E)$, then $f$ can be written as

$$
f(z)=\sum_{n=0}^{\infty} z^{n} a_{n} \quad a_{n} \in E, \quad z \in \Delta .
$$

A brief discussion on Banach space valued analytic functions can be found in [1].

Theorem 4.1. With the above notations $H(\Delta, E)$ is a complex unital Banach algebra with point wise addition, point wise multiplication and the norm given by

$$
\|f\|_{\infty}=\sup \{\|f(z)\|: z \in \Delta\} .
$$

Proof. In section 2, we observed that $C(\Delta, E)$ is a complex unital Banach algebra. Clearly $H(\Delta, E) \subseteq C(\Delta, E)$. Let $f, g \in H(\Delta, E)$ and $\alpha \in \mathbb{C}$. It is routine to check that $f+g, f g, \alpha f$ are analytic functions in $\Delta^{0}$. The unit element of $H(\Delta, E)$ is denoted by 1 and is defined as,

$$
1(z)=e \text { for all } z \in \Delta .
$$

Thus $H(\Delta, E)$ is a subalgebra of $C(\Delta, E)$. Next to show that it is a closed subalgebra, consider a sequence $f_{n} \in H(\Delta, E)$ and $f_{n} \rightarrow f$ in $C(\Delta, E)$. We show that $f \in H(\Delta, E)$. Let $\gamma$ be a rectifiable closed path in $\Delta^{0}$. By an analogue 
of Cauchy's theorem for Banach space valued analytic functions (Theorem 1.70 , [1]), we obtain

$$
\int_{\gamma} f(z) d z=\int_{\gamma} \lim _{n \rightarrow \infty} f_{n}(z) d z=\lim _{n \rightarrow \infty} \int_{\gamma} f_{n}(z) d z=0 .
$$

Hence by an analogue of Morera's theorem for Banach space valued functions [1], $f \in H(\Delta, E)$. Hence $H(\Delta, E)$ is a unital Banach algebra.

We call this algebra as the $E$ valued disk algebra. Now we consider an element $f \in H(\Delta, E)$ and investigate the relationship between the spectrum $\sigma(f)$ of $f$ and the spectra $\sigma(f(z))$ for $z \in \Delta$. We shall undertake similar study of pseudospectrum and condition spectrum. The main result depends on the following lemma, compare with Theorem 2.1.

Lemma 4.2. Let $f \in H(\Delta, E)$. Then $f$ is invertible in $H(\Delta, E)$ if and only if $f(z)$ is invertible in $E$ for all $z \in \Delta$. In this case $f^{-1}(z)=f(z)^{-1}$ for all $z \in \Delta$. In other words, $H(\Delta, E)$ is inverse-closed in $C(\Delta, E)$.

Proof. Let $f \in H(\Delta, E)$ be invertible with the inverse $g \in H(\Delta, E)$. Then

$$
f(z) g(z)=e=g(z) f(z) \text {. }
$$

This implies $f(z)$ is invertible in $E$ for all $z \in \Delta$.

Next assume that $f(z)$ is invertible in $E$ for all $z \in \Delta$. We claim that $f$ is invertible in $H(\Delta, E)$. Define $g: \Delta \rightarrow E$ by,

$$
g(z)=f(z)^{-1} \quad \text { for all } z \in \Delta .
$$

Then by Theorem $2.1 g \in C(\Delta, E)$. Also it is straightforward to show that $g$ is differentiable at each $z_{0} \in \Delta^{0}$ and

$$
g^{\prime}\left(z_{0}\right)=f\left(z_{0}\right)^{-1} f^{\prime}\left(z_{0}\right) f\left(z_{0}\right)^{-1} .
$$

Hence by definition $g \in H(\Delta, E)$.

Theorem 4.3. Let $f \in H(\Delta, E)$. Then

$$
\begin{aligned}
& \text { 1. } \sigma(f)=\bigcup_{z \in \Delta} \sigma(f(z)) \text {. } \\
& \text { 2. } \Lambda_{\epsilon}(f)=\bigcup_{z \in \Delta} \Lambda_{\epsilon}(f(z)) \text { for all } \epsilon>0 \text {. } \\
& \text { 3. } \sigma_{\epsilon}(f) \supseteq \bigcup_{z \in \Delta} \sigma_{\epsilon}(f(z)) \text { for all } 0<\epsilon<1 \text {. } \\
& \text { 4. } \sigma_{\epsilon}(f) \subseteq \bigcup_{z \in \Delta}\left\{D\left(\alpha, \epsilon_{1}\right): f(z)=\alpha e\right\} \bigcup_{z \in \Delta}\left\{\sigma_{\frac{\epsilon_{1}}{m_{z}}}(f(z)): m_{z}>0\right\}
\end{aligned}
$$

where $0<\epsilon<1, \epsilon_{1}=\frac{2 \epsilon}{1-\epsilon}\|f\|_{\infty}$ and for $z \in \Delta, m_{z}=\inf \{\|\lambda-f(z)\|: \lambda \in \mathbb{C}\}$.

Proof. Let $f \in H(\Delta, E)$. By Lemma 4.2,H( $H, E)$ is inverse-closed in $C(\Delta, E)$. It follows from Theorem 1.4 that $\sigma(f, H(\Delta, E))=\sigma(f, C(\Delta, E)), \Lambda_{\epsilon}(f, H(\Delta, E))=$ $\Lambda_{\epsilon}(f, C(\Delta, E))$ for all $\epsilon>0$ and $\sigma_{\epsilon}(f, H(\Delta, E))=\sigma_{\epsilon}(f, C(\Delta, E))$ for all $0<\epsilon<1$. 
Now (1) follows from Theorem 2.1, (2) follows from Theorem 2.2, (3) follows from Theorem 2.3 and (4) follows from Theorem 2.3.

\section{LOWER TRIANGULAR BLOCK TOEPLITZ OPERATORS WITH CONTINUOUS SYMBOLS}

In this section we describe the spectrum, pseudospectrum and condition spectrum of a lower triangular block Toeplitz operator with continuous symbol using the theory developed in the previous section. For this purpose we take $E=\left(\mathbb{C}^{m \times m},\|\cdot\|_{2}\right)$. If $\Phi \in H\left(\Delta, \mathbb{C}^{m \times m}\right)$, then $\Phi=\left[\Phi_{i j}\right]_{i, j=1}^{m}$ where each $\Phi_{i j}$ is a complex valued function that is continuous in $\Delta$ and analytic in $\Delta^{0}$. Note that if $T$ is a block Toeplitz operator with symbol $\Phi$, then $\Phi \in H\left(\Delta, \mathbb{C}^{m \times m}\right)$ if and only if $T$ is lower triangular.

Theorem 5.1. Let $A$ be the set of all lower triangular block Toeplitz operators on $\ell^{2}\left(\mathbb{N}, \mathbb{C}^{m}\right)$ with continuous symbols. Then $A$ is an inverse-closed Banach subalgebra of $B L\left(\ell^{2}\left(\mathbb{N}, \mathbb{C}^{m}\right)\right)$.

Proof. Let $T$ be a block Toeplitz operator on $\ell^{2}\left(\mathbb{N}, \mathbb{C}^{m}\right)$ with continuous symbol $\Phi(t), t \in \Delta$. For $U, R \in B L\left(\ell^{2}\left(\mathbb{N}, \mathbb{C}^{m}\right)\right), S=U R$ is defined as,

$$
S_{i j}=\sum_{k=1}^{\infty} U_{i k} R_{k j} .
$$

Thus the elementary calculation shows that the inverse of a lower triangular block Toeplitz operator is a lower triangular block Toeplitz operator. Thus $T^{-1}$ is a lower triangular block Toeplitz operator with symbol $\Phi(t)^{-1}, t \in \Delta$. Since $\Phi(t) \in C\left(\Gamma, \mathbb{C}^{m \times m}\right)$ we have $\Phi(t)^{-1} \in C\left(\Gamma, \mathbb{C}^{m \times m}\right)$. Hence $T$ is invertible in A.

Theorem 5.2. Let $T$ be a lower triangular block Toeplitz operator on $\ell^{2}\left(\mathbb{N}, \mathbb{C}^{m}\right)$ with symbol $f \in H\left(\Delta, \mathbb{C}^{m \times m}\right)$. Then $\sigma(T)=\sigma(f)$. Also for each $\epsilon>0, \Lambda_{\epsilon}(T)=$ $\Lambda_{\epsilon}(f)$ and for $0<\epsilon<1, \sigma_{\epsilon}(T)=\sigma_{\epsilon}(f)$.

Proof. Let $A$ be the Banach algebra of all lower triangular block Toeplitz operators with continuous symbol on $\ell^{2}\left(\mathbb{N}, \mathbb{C}^{m}\right)$ and $B=H\left(\Delta, \mathbb{C}^{m \times m}\right)$. First note that $A$ is inverse closed in $B L\left(\ell^{2}\left(\mathbb{N}, \mathbb{C}^{m}\right)\right)$. Hence by Theorem 1.4 the spectrum of $T$ as an element of $A$ is the same as the spectrum of $T$ regarded as an element of $B L\left(\ell^{2}\left(\mathbb{N}, \mathbb{C}^{m}\right)\right)$. The same can be said about the pseudospectrum and condition spectrum. The map $\Phi: A \rightarrow B$ defined by,

$$
\Phi(T)=\text { symbol of } T
$$

is linear, bijective, unital, multiplicative(Lemma 4.1, page 575 of [8]) and isometry(Corollary 3.2, page 573 of [8]). Hence the result follows from Theorem 1.5. 
Corollary 5.3. Let $T$ be a lower triangular block Toeplitz operator on $\ell^{2}\left(\mathbb{N}, \mathbb{C}^{m}\right)$ with symbol $f=\left[f_{k l}\right]_{k, l=1}^{m} \in H\left(\Delta, \mathbb{C}^{m \times m}\right)$. Then

1. $\sigma(T)=\bigcup_{z \in \Delta} \sigma(f(z))=\bigcup_{z \in \Delta} \sigma\left(\left[f_{k l}(z)\right]_{k, l=1}^{m}\right)$.

2. $\Lambda_{\epsilon}(T)=\bigcup_{z \in \Delta} \Lambda_{\epsilon}(f(z))=\bigcup_{z \in \Delta} \Lambda_{\epsilon}\left(\left[f_{k l}(z)\right]_{k, l=1}^{m}\right)$ for all $\epsilon>0$.

3. $\sigma_{\epsilon}(T) \supseteq \bigcup_{z \in \Delta} \sigma_{\epsilon}(f(z))=\bigcup_{z \in \Delta} \sigma_{\epsilon}\left(\left[f_{k l}(z)\right]_{k, l=1}^{m}\right)$ for all $0<\epsilon<1$.

4. $\sigma_{\epsilon}(T) \subseteq \bigcup_{z \in \Delta}\left\{D\left(\alpha, \epsilon_{1}\right):\left[f_{k l}(z)\right]_{k, l=1}^{m}=\alpha I_{m}\right\} \bigcup_{z \in \Delta}\left\{\sigma_{\frac{\epsilon_{1}}{m_{z}}}\left(\left[f_{k l}(z)\right]_{k, l=1}^{m}\right): m_{z}>0\right\}$.

where $I_{m}$ denotes the $m \times m$ identity matrix, $0<\epsilon<1, \epsilon_{1}=\frac{2 \epsilon}{1-\epsilon}\|f\|_{\infty}$ and for $z \in \Delta, m_{z}=\inf \{\|\lambda-f(z)\|: \lambda \in \mathbb{C}\}$.

Proof. (1) From Theorem 5.2 we have

$$
\sigma(T)=\sigma(f)=\bigcup_{z \in \Delta} \sigma(f(z)) .
$$

Since $f \in H\left(\Delta, \mathbb{C}^{m \times m}\right), f=\left[f_{k l}\right]_{k, l=1}^{m}$ and each $f_{k l} \in H(\Delta, \mathbb{C})$ (i.e, each $f_{k l}$ is a complex analytic function). Hence

$$
\bigcup_{z \in \Delta} \sigma(f(z))=\bigcup_{z \in \Delta} \sigma\left(\left[f_{k, l}(z)\right]_{k, l=1}^{m}\right)
$$

(2) Let $\epsilon>0$. From Theorem 5.2 we have

$$
\Lambda_{\epsilon}(T)=\Lambda_{\epsilon}(f)=\bigcup_{z \in \Delta} \Lambda_{\epsilon}(f(z))=\bigcup_{z \in \Delta} \Lambda_{\epsilon}\left(\left[f_{k, l}(z)\right]_{k, l=1}^{m}\right) .
$$

(3) Let $0<\epsilon<1$. From Theorem 5.2 we have

$$
\sigma_{\epsilon}(T)=\sigma_{\epsilon}(f) \supseteq \bigcup_{z \in \Delta} \sigma_{\epsilon}(f(z))=\bigcup_{z \in \Delta} \sigma_{\epsilon}\left(\left[f_{k, l}(z)\right]_{k, l=1}^{m}\right) .
$$

(4) Let $0<\epsilon<1$. From Theorem 5.2 we have

$$
\sigma_{\epsilon}(T)=\sigma_{\epsilon}(f) \subseteq \bigcup_{z \in \Delta}\left\{D\left(\alpha, \epsilon_{1}\right): f(z)=\alpha I_{m}\right\} \bigcup_{z \in \Delta}\left\{\sigma_{\frac{\epsilon_{1}}{m_{z}}}(f(z)): m_{z}>0\right\}
$$

where $\epsilon_{1}=\frac{2 \epsilon}{1-\epsilon}\|f\|_{\infty}$ and for $z \in \Delta, m_{z}=\inf \{\|\lambda-f(z)\|: \lambda \in \mathbb{C}\}$. We note that the right hand side is equal to

$$
\bigcup_{z \in \Delta}\left\{D\left(\alpha, \epsilon_{1}\right):\left[f_{k, l}(z)\right]_{k, l=1}^{m}=\alpha I_{m}\right\} \bigcup_{z \in \Delta}\left\{\sigma_{\frac{\epsilon_{1}}{m_{z}}}\left(\left[f_{k, l}(z)\right]_{k, l=1}^{m}\right): m_{z}>0\right\}
$$


Corollary 5.4. Let $T$ be a lower triangular Toeplitz operator on $\ell^{2}$ with symbol $f \in H(\Delta)$. Then

1. $\sigma(T)=\{f(\lambda): \lambda \in \Delta\}=f(\Delta)$.

2. $\Lambda_{\epsilon}(T)=\bigcup\{D(f(\lambda), \epsilon): \lambda \in \Delta\}$ for all $\epsilon>0$.

3. $\sigma_{\epsilon}(T) \supseteq\{(f(\lambda): \lambda \in \Delta\}$ for all $0<\epsilon<1$.

4. $\sigma_{\epsilon}(T) \subseteq \bigcup\left\{D\left(f(\lambda), \frac{2 \epsilon}{1-\epsilon}\|f\|_{\infty}\right): \lambda \in \Delta\right\}$ for all $0<\epsilon<1$.

Where $\Delta$ is the closed unit disk in the complex plane.

Proof. (1) From Corollary 5.3 we have

$$
\sigma(T)=\sigma(f)=\bigcup_{\lambda \in \Delta} \sigma(f(\lambda))=\bigcup_{\lambda \in \Delta} f(\lambda)=f(\Delta) .
$$

(2) Let $\epsilon>0$. From Corollary 5.3 we have

$$
\Lambda_{\epsilon}(T)=\Lambda_{\epsilon}(f)=\bigcup_{\lambda \in \Delta} \Lambda_{\epsilon}(f(\lambda))=\bigcup_{\lambda \in \Delta} D(f(\lambda), \epsilon)
$$

(3) Let $0<\epsilon<1$. From Corollary 5.3 we have

$$
\sigma_{\epsilon}(T)=\sigma_{\epsilon}(f) \supseteq \bigcup_{\lambda \in \Delta} \sigma_{\epsilon}(f(\lambda))=\bigcup_{\lambda \in \Delta}\{f(\lambda)\}=\{f(\lambda): \lambda \in \Delta\} .
$$

(4) Let $0<\epsilon<1$. From Corollary 5.3 we have

$$
\sigma_{\epsilon}(T)=\sigma_{\epsilon}(f) \subseteq \bigcup_{\lambda \in \Delta} D\left(f(\lambda), \frac{2 \epsilon}{1-\epsilon}\|f\|_{\infty}\right) .
$$

A wealth of information about spectra of Toeplitz operators with symbols of different kind can be found in [3]. Pseudospectra of Toeplitz operators are discussed in the article [4].

Remark 5.5. Theorem 5.2 and its corollaries are useful in computing spectrum $\sigma(T)$, pseudospectrum $\Lambda_{\epsilon}(T)$ and condition spectrum $\sigma_{\epsilon}(T)$ of a lower triangular block Toeplitz operator $T$ with a continuous symbol $f \in H\left(\Delta, \mathbb{C}^{m \times m}\right)$. Since for each $z \in \Delta, f(z)$ is a square matrix of order $m$, we can compute and plot $\sigma(f(z)), \Lambda_{\epsilon}(f(z)), \sigma_{\epsilon}(f(z))$ for various values of $z \in \Delta$. Then the respective unions of these sets are approximation of $\sigma(T), \Lambda_{\epsilon}(T), \sigma_{\epsilon}(T)$.

The situation is very satisfactory when $m=1$, that is $T$ is a lower triangular Toeplitz operator on $\ell^{2}$ with a continuous symbol $f$. Then from Corollary 5.4, $\sigma(T)=f(\Delta)$ is a compact set in the complex plane say $D . \Lambda_{\epsilon}(T)$ is the compact set that contains $D$ and all points in the complex plane at a distance less that or equal to $\epsilon$ from $D$. From Theorem 5.2, $\sigma_{\epsilon}(T)=\sigma_{\epsilon}(f)$. We can consider certain number of uniformly distributed points in the $\operatorname{disc}\left\{z \in \mathbb{C}:|z| \leq \frac{1+\epsilon}{1-\epsilon}\|f\|_{\infty}\right\}$ [11], evaluate $\|z-f\|_{\infty}\left\|(z-f)^{-1}\right\|_{\infty}$ at each of these points and include and 
save those $z$ for which $\|z-f\|_{\infty}\left\|(z-f)^{-1}\right\|_{\infty} \geq \frac{1}{\epsilon} \cdot \sigma_{\epsilon}(T)$ is also contained in a similar set as pseudospectra. For example, if $L$ is the right shift operator on $\ell^{2}$ then $f(z)=z, \sigma(T)=\Delta, \Lambda_{\epsilon}(T)$ is the closed disk $\{\lambda \in \mathbb{C}:|\lambda| \leq 1+\epsilon\}, \sigma_{\epsilon}(T)$ is the closed disk $\left\{\lambda \in \mathbb{C}:|\lambda| \leq \frac{1+\epsilon}{1-\epsilon}\right\}$ (see Example 2.14 in [11]).

Remark 5.6. We can develop a similar theory in case of upper triangular block Toeplitz operators with continuous symbols.

Example 5.7. Consider the lower triangular block Toeplitz operator

$$
T=\left[\begin{array}{cccccc}
{\left[\begin{array}{ll}
1 & 2 \\
0 & 1
\end{array}\right]} & {[0]} & {[0]} & {[0]} & \ldots & . \\
{\left[\begin{array}{ll}
0 & -1 \\
3 & -2
\end{array}\right]} & {\left[\begin{array}{ll}
1 & 2 \\
0 & 1
\end{array}\right]} & {[0]} & {[0]} & \cdots & . \\
{[0]} & {\left[\begin{array}{ll}
0 & -1 \\
3 & -2
\end{array}\right]} & {\left[\begin{array}{ll}
1 & 2 \\
0 & 1
\end{array}\right]} & {[0]} & . . & . \\
{[0]} & {[0]} & {\left[\begin{array}{ll}
0 & -1 \\
3 & -2
\end{array}\right]} & {\left[\begin{array}{ll}
1 & 2 \\
0 & 1
\end{array}\right]} & . . & . \\
. & . & . & . & \ldots & . \\
. & . & . & . & \ldots & .
\end{array}\right]
$$

where [0] denotes the $2 \times 2$ zero matrix. Let $f$ denote the symbol of $T$. Then

$$
f(x)=\left[\begin{array}{ll}
f_{11}(x) & f_{12}(x) \\
f_{21}(x) & f_{22}(x)
\end{array}\right]=\left[\begin{array}{cc}
1 & 2-x \\
3 x & 1-2 x
\end{array}\right], x \in \Delta .
$$

By Corollary 5.3, $\sigma(T)$ is equal to the union of the eigenvalues of the matrix $f(x)$ for $x \in \Delta$. The $\epsilon$-pseudospectrum and $\epsilon$-condition spectrum also can be calculated as explained in Remark 5.5. The following figures are obtained using matlab. Figure 3.1 shows $\sigma(T)$, Figure 3.2 shows $\Lambda_{0.5}(T)$, Figure $3.3\left(D_{1}\right)$ shows the set defined in (3) of Corollary 5.3 and Figure $3.4\left(D_{2}\right)$ shows the set defined in (4) of Corollary 5.3 for $\epsilon=0.01$. We have $D_{1} \subseteq \sigma_{0.01}(T) \subseteq D_{2}$, Figure 3.5 shows $\sigma_{0.01}(T)$.
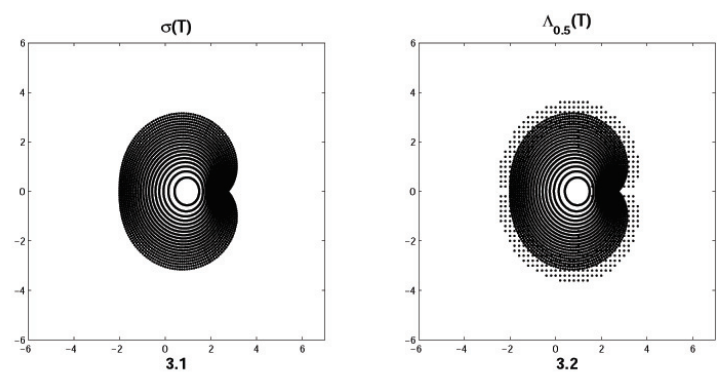

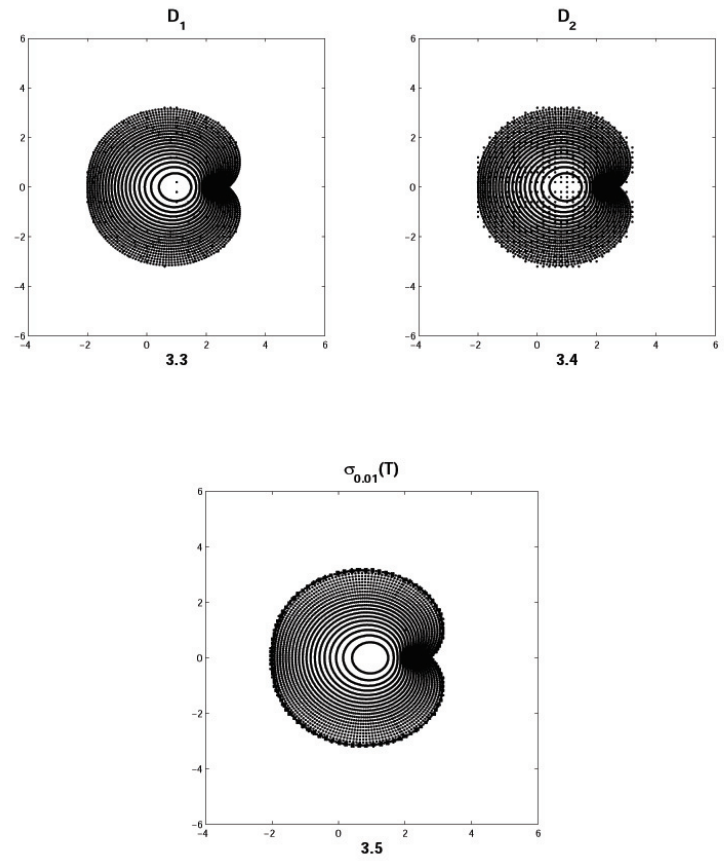

Example 5.8. Consider the lower triangular block Toeplitz operator

$$
T_{1}=\left[\begin{array}{cccccc}
{\left[\begin{array}{ll}
1 & 2 \\
0 & 1
\end{array}\right]} & {[0]} & {[0]} & {[0]} & \ldots & . \\
{\left[\begin{array}{ll}
1 & 0 \\
0 & 0
\end{array}\right]} & {\left[\begin{array}{ll}
1 & 2 \\
0 & 1
\end{array}\right]} & {[0]} & {[0]} & \ldots & . \\
{[0]} & {\left[\begin{array}{ll}
1 & 0 \\
0 & 0
\end{array}\right]} & {\left[\begin{array}{ll}
1 & 2 \\
0 & 1
\end{array}\right]} & {[0]} & . . & . \\
{[0]} & {[0]} & {\left[\begin{array}{ll}
1 & 0 \\
0 & 0
\end{array}\right]} & {\left[\begin{array}{cc}
1 & 2 \\
0 & 1
\end{array}\right]} & . . & . \\
. & . & . & . & \ldots & . \\
. & . & . & . & \ldots & .
\end{array}\right]
$$

where [0] denotes the $2 \times 2$ zero matrix. Let $f$ denote the symbol of $T_{1}$. Then

$$
f(x)=\left[\begin{array}{ll}
f_{11}(x) & f_{12}(x) \\
f_{21}(x) & f_{22}(x)
\end{array}\right]=\left[\begin{array}{cc}
1+x & 2 \\
0 & 1
\end{array}\right], x \in \Delta .
$$

The following figures are obtained using matlab. Figure 4.1 shows $\sigma\left(T_{1}\right)$, Figure 4.2 shows $\Lambda_{0.5}\left(T_{1}\right)$, Figure $4.3\left(D_{1}\right)$ shows the set defined in (3) of Corollary 5.3 and Figure 4.4 $\left(D_{2}\right)$ shows the set defined in (4) of Corollary 5.3 for $\epsilon=0.01$. We have $D_{1} \subseteq \sigma_{0.01}\left(T_{1}\right) \subseteq D_{2}$, Figure 4.5 shows $\sigma_{0.01}\left(T_{1}\right)$.

\section{REFERENCES}

1. Y.A. Abramovich and C.D. Aliprantis, An invitation to operator theory, Graduate Studies in Mathematics, 50, Amer. Math. Soc., Providence, RI, 2002.

2. F.F. Bonsall and J. Duncan, Complete normed algebras, Springer, New York, 1973. 

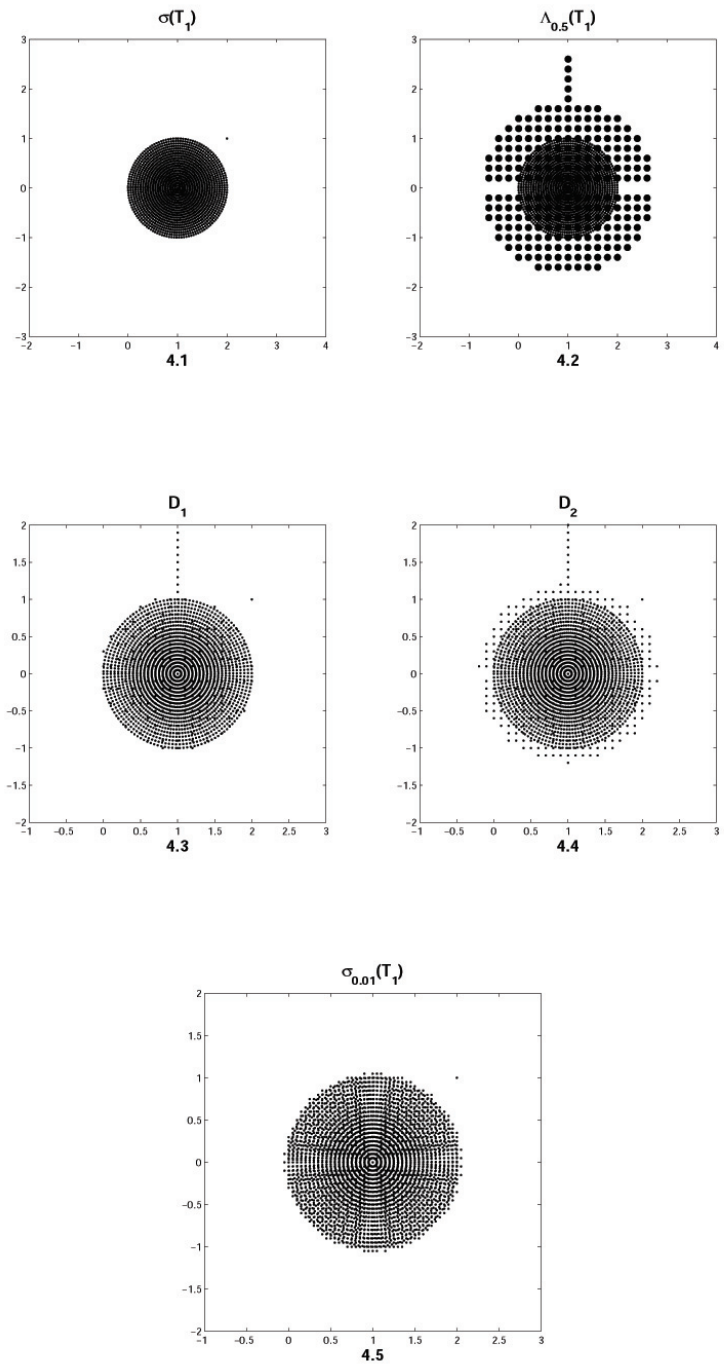

3. A. Böttcher and B. Silbermann, Analysis of Toeplitz operators, second edition, Springer Monographs in Mathematics, Springer, Berlin, 2006.

4. A. Böttcher, Pseudospectra and singular values of large convolution operators, J. Integral Equations Appl. 6 (1994), no. 3, 267-301.

5. I. Feldman, Finiteness of the discrete spectrum of some block Toeplitz operators Integral Equations Operator Theory 16 (1993), no. 3, 385-391.

6. R. Hagen, S. Roch and B. Silbermann, $C^{*}$-algebras and numerical analysis, Monographs and Textbooks in Pure and Applied Mathematics, 236, Dekker, New York, 2001.

7. I. Gohberg, S. Goldberg and M.A. Kaashoek, Basic classes of linear operators, Birkhäuser, Basel, 2003.

8. I. Gohberg, S. Goldberg and M.A. Kaashoek, Classes of linear operators. Vol. II, Operator Theory: Advances and Applications, 63, Birkhäuser, Basel, 1993.

9. I. Gohberg and M.A. Kaashoek, Projection method for block Toeplitz operators with operator-valued symbols Oper. Theory Adv. Appl. 71 (1994), 79-104.

10. G. Krishna Kumar and S.H. Kulkarni, Linear maps preserving pseudospectrum and condition spectrum, Banach J. Math. Anal. 6 (2012), no. 1, (45-60).

11. S.H. Kulkarni and D. Sukumar, The condition spectrum, Acta Sci. Math. (Szeged) 74 (2008), no. 3-4, 625-641. 
12. A. Lumsdaine and D. Wu, Spectra and pseudospectra of block Toeplitz matrices, Linear Algebra Appl. 272 (1998), 103-130.

13. L. Reichel and L.N. Trefethen, Eigenvalues and pseudo-eigenvalues of Toeplitz matrices, Linear Algebra Appl. 162/164 (1992), 153-185.

14. S. Roch, P.A. Santos and B. Silbermann, Non-commutative Gelfand theories, Universitext, Springer, London, 2011.

15. A. Rogozhin, The singular value behaviour of finite sections of block Toeplitz operators SIAM J. Matrix Anal. Appl. 27 (2005), no. 1, 273-293.

16. W. Rudin, Functional analysis, Second edition, McGraw-Hill, New York, 1991.

17. L.N. Trefethen and M. Embree, Spectra and pseudospectra, Princeton Univ. Press, Princeton, NJ, 2005.

Department of Mathematics, Indian Institute of Technology Madras, Chennai600 036, INDIA.

E-mail address: krishna.math@gmail.com

E-mail address: shk@iitm.ac.in 